\title{
Distributed Detection of Node Replication Attacks Resilient to Many Compromised Nodes in Wireless Sensor Networks
}

\author{
Yuichi Sei \\ The University of Tokyo 7-3-1 Hongo, \\ Bunkyo-ku, Tokyo 113-8654, Japan \\ sei@nii.ac.jp
}

\author{
Shinichi Honiden \\ National Institute of Informatics \\ 2-1-2 Hitotsubashi, Chiyoda-ku, Tokyo 101-8430, \\ Japan \\ The University of Tokyo 7-3-1 Hongo, \\ Bunkyo-ku, Tokyo 113-8654, Japan \\ honiden@nii.ac.jp
}

\begin{abstract}
In large-scale sensor networks, sensor nodes are at high risk of being captured and compromised. Once a sensor node is compromised, all the secret keys, data, and code stored on it are exposed to the attacker. The attacker can insert arbitrary malicious code in the compromised node. Moreover, he can easily replicate it in a large number of clones and deploy them on the network. This node replication attack can form the basis of a variety of attacks such as DoS attacks, and Sybil attacks. Previous studies of node replication attacks have had some drawbacks; they need a central trusted entity or they become vulnerable when many nodes are compromised. Therefore, we propose a distributed protocol for detecting node replication attacks that is resilient to many compromised nodes. Our method does not need any reliable entities and has a high detection rate of replicated nodes. Our analysis and simulations demonstrate our protocol is effective even when there are a large number of compromised nodes.

Keywords
\end{abstract}

Security, Algorithm, Wireless sensor networks, Node compromising attack, Node replication attack

\section{INTRODUCTION}

A wireless sensor network consists of a large number of low-cost and low-power sensors. The sensor nodes collect information about their environment. Such a network is suitable for military surveillance, forest fire monitoring, etc. To join the network, new nodes should pass certain authentication phases of neighbor discovery protocols $[3,7]$. These protocols determine whether the new nodes are legitimate or not by checking their IDs and corresponding keys. A problem is that attackers may capture and compromise sensors. They can obtain the secret keys stored in the compromised nodes and insert arbitrary malicious code in the nodes. Moreover, they can replicate a large number of clones and deploy them to the network. Because these replicated nodes have legitimate IDs and corresponding legitimate keys, neighbor discovery protocols cannot detect them.

Copyright 2008 ICST 978-963-9799-36-3.

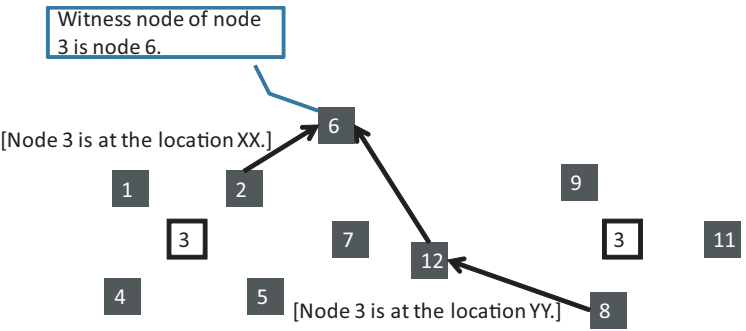

Figure 1: Replicated nodes and witness node (the numbers represent the node IDs)

Related studies [5, 15, 23] have proposed several protocols to detect node replication attacks. The basic idea is as follows. Every node broadcasts its node ID and location to one-hop neighbors. We call this message a claim and the node that broadcasts a claim a claimer node. The neighbors of the claimer node forward this claim toward a witness node. A witness node is determined by the node ID of the claimer node. If the witness node receives more than one claim containing the same node ID but from different locations, the witness node can conclude that more than one node with the same node ID exists (Fig. 1).

RED [5] can detect replicated nodes efficiently, but it needs a trusted entity (e.g., the base station). We cannot assume all wireless sensor networks have a trusted entity. For example, when a sensor network is deployed in a hostile environment such as a battlefield, the base station is an attractive target to the attackers. In this paper, we propose a protocol which does not need any trusted entities. B. Parno et.al. proposed two distributed detection protocols [15]. First, Randomized Multicast (RM) distributes node location information to randomly-selected witnesses. Second, Line-Selected Multicast (LSM) is an efficient distributed detection protocol. However, both have relatively low detection rates. SDC and P-MPC [23] are other distributed detection protocols. They do not require trusted entities and can detect replicated nodes with high probability. However, if an attacker compromises many nodes, the detection rates will greatly decrease. Our goals is to propose a replicated node detection protocol which does not need any trusted entities and is resilient to many compromised nodes attacks.

The rest of this paper is organized as follows. Section 2 presents a model sensor network and describes node replication attacks. Sec- 
tion 3 discusses the related methods and their problems. Section 4 presents the design of our algorithm of replicated node detection. In Section 5, we evaluate our method by using a mathematical analysis and simulations. Section 6 concludes the paper.

\section{SYSTEM MODEL}

In this section, we define our assumed sensor network model and the model of node replication attacks.

\subsection{Sensor Network Model}

We take into account a sensor network composed of a large number of small sensor nodes. Since they are designed to be inexpensive, the sensor nodes are assumed not to be equipped with tamperresistant hardware. Once deployed, each node can determine its geographic location by using a localization scheme $[2,19]$.

The compromised nodes can launch many attacks. However, these threats are addressed in other research. We assume that sensor nodes can detect an attack involving dropping and fabricating messages by using detecting protocols such as $[12,22]$ and further assume that nodes cannot alter their IDs by detecting false IDs protocols [13].

These assumptions are the same as in other replicated node detection protocols $[5,15,23]$.

We do not assume the existence of a trusted entity. In military applications, sensor nodes may be deployed in hostile regions. A base station would be an attractive target to the attackers. Many studies on wireless sensor networks devised various protocols that do not need trusted entities $[11,6]$. The sensor nodes are loosely time synchronized as in $\mu$ TESLA [16].

\subsection{Node Replication Attack}

An attacker may compromise multiple sensor nodes in a network. Once a sensor node is compromised, all the secret keys, data, and code stored on it are exposed to the attacker. The attacker can easily replicate it in a large number of clones and deploy them to the network. This node replication attack can be the basis for launching a variety of attacks such as DoS attacks [21, 14] and Sybil attacks [13]. If there are many replicated nodes in the network, they can multiply the damage to the network. Therefore, we should quickly detect replicated nodes.

\section{RELATED WORK}

We introduce five protocols: RED [5], RM and LSM [15], and SDC and P-MPC [23]. First, we explain their common method and goal. In all of these protocols, each node is preloaded with a unique node ID, a shared function which calculates the location of a witness node of an arbitrary node from the node ID, and a private key for creating a signature. They use ID-based public key crypto-schemes $[18,1]$ for creating and checking signatures. Each node can easily calculate a corresponding public key for a given ID. However, we cannot calculate the private key for an arbitrary ID because of its computationally complexity. When a detection process starts, each node $n_{i}$ sends its node ID $i$, location, and signature to one-hop neighbor nodes (referred to as a claim). The neighbor nodes create a corresponding public key for the node ID $i$; then it checks the correctness of the signature. Next, each node calculates the location of the witness node for $n_{i}$. They forward the received claim toward the witness node by using a geographic routing protocol such as GPSR [8] with probability $p$. We call the node which forwards the claim a reporter node. If the witness node receives several claims that contain the same node ID but different locations, the witness node concludes that these sending nodes have been compromised and that an attacker replicated them.

If the location of the witness node is statically determined by the given node ID, an attacker who compromised one node can specify the witness nodes of all nodes. For example, if an attacker compromises a node and obtains a function that determines the location of the witness node from a given ID, he can compromise the witness node. Then the attacker can create and deploy a large number of replicated nodes without detection. Therefore, we need to avoid specifying the witness node from an attacker even if the attacker compromised many nodes.

In the RED protocol [5], a trusted entity broadcasts a one-time seed to the whole network. The location of the witness node of a node is determined from the node ID and the seed. Because the seed changes every time, an attacker cannot specify the location of a witness node in advance. The authors of RED said one can also use distributed protocol without a trusted entity such as a local leader election mechanism [4] to create a one-time seed. However, the authors did not mention how to create it; moreover, the local leader election mechanism creates a local leader from a small number of sensor nodes. Even worse, the method does not consider the existence of compromised nodes. Therefore, we cannot use it to create a global leader of a sensor network composed of a large number of nodes with some of them compromised.

In the RM and LSM protocols [15], the neighbor nodes of node $n_{i}$ determine several witness nodes randomly. By exploiting the birthday paradox, at least one node is likely to receive conflicting location claims for a particular node. In the LSM protocol, each node which forwards claims also saves the claim. That is, the forwarding nodes are also witness nodes of a node which has the node ID in a claim. Therefore, LSM gives a higher detection rate than that of RM. However, both protocols have relatively lower detection rates compared with RED.

In the SDC and P-MPC protocols [23], the sensor network is considered to be a geographic grid. In the SDC protocol, witness nodes candidates of one node are all nodes of a grid. The grid is statically determined by the node ID, but which nodes in the grid actually become witness nodes is determined randomly. In P-MPC, to increase resiliency to many compromised nodes, the candidate witness nodes for one node are all nodes of several grids (three grids by default). The number of nodes in a grid was set to 100 by default. If an attacker compromises a node, learns the witness grids of the node, and compromises 100 nodes of one of the grids, the detection rate will decrease to about $1 / 8$ (see the evaluation in section 5). Moreover, if the attacker compromises all nodes of the three grids (i.e., 300 nodes), these protocols can never detect node replication attacks. Many recent studies on security in wireless sensor networks have proposed methods that are resilient to many nodes being compromised (e.g., a thousand compromised nodes) $[17,10$, $9,20]$. Therefore, we need to propose a replicated node detection protocol resilient to many compromised nodes.

\section{DISTRIBUTED DETECTION PROTOCOL}

This section presents our design for detecting replicated nodes.

\subsection{Overview}


To prevent an attacker from learning the location of a witness node of a compromised node, our protocol uses a one-time seed for each replicated node detection process. In the RED protocol [5], a trusted entity gives it to all the nodes. In our protocol, each node sends a one-time seed to other nodes in turn. That is, each node has the role of starting a detection process and it is preloaded with the assigned turn number and seed for the turn.

When node $n_{i}$ has a turn starting detection process, it sends the seed and its ID with a signature. Other nodes verify the signature and execute the detection process if the verification succeeds. Here, we should consider that sensor nodes break down easily and an attacker may compromise a large number of nodes. We divide nodes into groups to increase resiliency to fault nodes and compromised nodes. The role of the starting detection process is not assigned to each node but to each group.

We assign each node its node ID and its role ID and group nodes according to their role IDs. The reason why we introduce role IDs is to prevent attackers from learning the locations of the node that has a turn starting detection process. Attackers can learn a node ID and a location of the corresponding node because each claim contains a location of a node and its node ID. Therefore, nodes should use role IDs for starting detection process. $n_{i, j}$ denotes a node whose node ID is $i$ and role ID is $j$.

In each group, nodes are sorted according to their role IDs. The number of nodes of a group is $N_{p}$. When a group $G_{i}$ has a turn starting detection process (the turn is calculated from the elapsed time and the time interval of detection processes $\Delta$ ), the first node of the group sends the seed and its role ID with its signature. If the process has not started after an internal interval time of detection process $\delta$ because of a failure, compromised node, or other reason, the second node of the group sends the seed and its role ID with its signature to the first node's place (see Fig. 2). Note that $N_{p} \cdot \delta$ can be less than but not greater than $\Delta$.

If at least one node of a group survives, the group can start the detection process during its turn. An attacker must compromise the first node of a group which has the next turn starting detection process if he wants to learn the location of the witness node in the next detection process.

The following subsections describe the details of our proposal. We use the notation presented in Table 1.

\subsection{Preparation of sensor nodes}

We preload each node $n_{i, j}$ with a hash function $H$, a time interval of detection processes $\Delta$, an internal time interval of detection process $\delta$, a function that calculates a location of a witness node from a seed and a node ID $F_{L}$, a function that calculates a public key for node IDs $F_{E}$, a private key for node ID $K_{i}^{-1}$, a function that calculates a public key for role IDs $\hat{F}_{E}$, and a private key for role ID $\hat{K}_{j}^{-1}$.

\subsection{Tasks of node $n_{i, j}$}

- Calculate the current turn number at fixed intervals of $\delta$. Let the reference time be $t_{s}$ and the current time be $t_{\text {current }}$. The current turn number is $T_{c}=\left\lfloor\left(t_{\text {current }}-t_{s}\right) / \Delta\right\rfloor$. The current internal turn number is $t_{c}=\left\lfloor\left[\left(t_{\text {current }}-t_{s}\right) \% \Delta\right] / \delta\right\rfloor$.

- Calculate the role ID of the node that will start the detection process during this internal turn. The role ID is $R_{I D}=T_{c}$

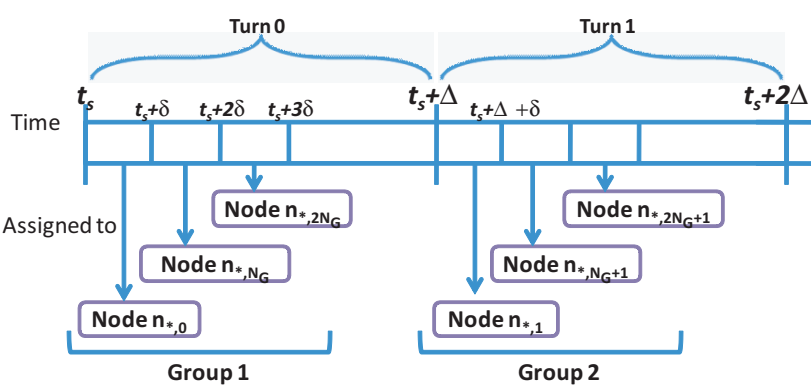

(a) Role of the starting detection process is assigned to each group $\left(N_{p}=3\right.$. '*' represents an arbitrary node ID.)

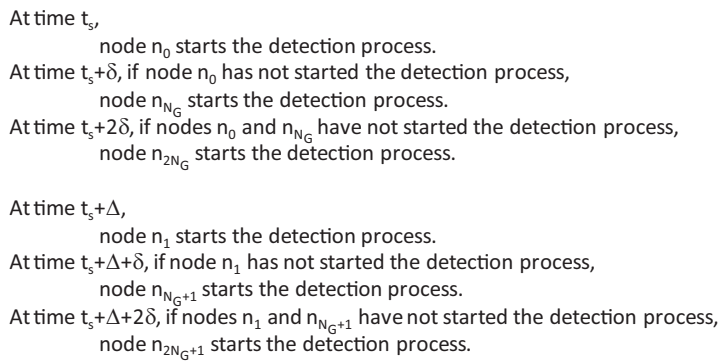

(b) Tasks of each node

Figure 2: Overview of our protocol

$\bmod N_{G}+t_{c} \cdot N_{G}$.

- If $j=R_{I D}$ and if in this turn $T_{c}$, the detection process has not been started, node $n_{i}$ starts process A.

- If node $n_{i}$ receives message $M_{A}$, it starts process $\mathrm{B}$.

- If node $n_{i}$ receives message $M_{A}^{\prime}$, it starts process C'.

\subsubsection{Process A}

- Node $n_{i}$ broadcasts a message

$$
M_{A}(i)=\left\langle i, l_{i}, S_{K_{i}^{-1}}\left(H\left(T_{c}\|i\| l_{i}\right)\right)\right\rangle
$$

to one-hop neighbor nodes where $l_{i}$ represents the location of node $n_{i}$. The symbol $\|$ represents a stream concatenation.

- Node $n_{i}$ then broadcasts a message

$$
M_{A_{2}}=\left\langle S_{\hat{K}_{R_{I D}^{-1}}^{-1}}\left(H\left(T_{c}\right)\right)\right\rangle
$$

to one-hop neighbor nodes. This value is the seed of the detection process in this turn.

\subsubsection{Process $B$}

Assume that node $n_{i}$ has received a message $M_{A}(j)$.

- Node $n_{i}$ verifies the location $l_{j}$. 


\section{Table 1: Notation}

\begin{tabular}{|c|c|}
\hline$N$ & Number of nodes in the network \\
\hline$n_{i}$ & Sensor node whose node ID is $i$ \\
\hline$n_{i, j}$ & $\begin{array}{l}\text { Sensor node whose node ID is } i \text { and role ID is } j \\
\text { (We can identify a node by node ID or role ID only.) }\end{array}$ \\
\hline$K_{a}$ & $\begin{array}{l}\text { Public key of a node whose node ID is } a \\
\text { (calculated by a function } F_{E}(a) \text { ) }\end{array}$ \\
\hline$K_{a}^{-1}$ & Private key of a node whose node ID is $a$ \\
\hline$\hat{K}_{a}$ & $\begin{array}{l}\text { Public key of a node whose role ID is } a \\
\text { (calculated by a function } \hat{F}_{E}(a) \text { ) }\end{array}$ \\
\hline$\hat{K}_{a}^{-1}$ & Private key of a node whose node ID is $a$ \\
\hline$N_{G}$ & Number of groups \\
\hline$N_{p}$ & Number of nodes per group $\left(N=N_{G} \cdot N_{p}\right)$ \\
\hline$C_{n}$ & Number of compromised nodes \\
\hline$S_{K}(M)$ & Signature of string $M$ by key $K$ \\
\hline$F_{L}(s, i)$ & $\begin{array}{l}\text { Location of witness node } \\
\text { obtained from seed } s \text { and node ID } i\end{array}$ \\
\hline$H(M)$ & Hash of $M$ \\
\hline$\Delta$ & Time interval of detection processes \\
\hline$\delta$ & Internal time interval of detection process \\
\hline & Average number of reporter nodes of one claimer no \\
\hline
\end{tabular}

- If node $n_{i}$ does not know the value of $S_{\hat{K}_{R_{I D}}^{-1}}\left(H\left(T_{c}\right)\right)$, it starts process B'. If node already knows the value of $S_{\hat{K}_{R_{I D}}^{-1}}\left(H\left(T_{c}\right)\right)$ or the verification in process B' succeeds, it starts process $\mathrm{C}$.

- If node $n_{i}$ succeeds with the verification in process B', it starts process A only once.

\subsubsection{Process B'}

- Node $n_{i}$ receives a message $M_{A_{2}}$ from node $n_{j}$.

- Node $n_{i}$ calculates the public key of $R_{I D} ; \hat{K}_{R_{I D}}=\hat{F}_{E}\left(R_{I D}\right)$ and calculates $H\left(T_{c}\right)$. Then it verifies $M_{A_{2}}$.

\subsubsection{Process $C$}

Assume that node $n_{i}$ has received a message $M_{A}(j)$.

- Node $n_{i}$ calculates the location of the witness node of node $n_{j}$

$$
l_{d s t}=F_{L}\left(S_{\hat{K}_{R_{I D}}^{-1}}\left(H\left(T_{c}\right)\right), j\right) .
$$

- Node $n_{i}$ forwards a message

$$
M_{A}^{\prime}(j)=\left\langle j, l_{j}, l_{d s t}, S_{K_{j}^{-1}}\left(H\left(T_{c}\|j\| l_{j}\right)\right)\right\rangle
$$

to an applicable node.

\subsubsection{Process $C^{\prime}$}

Assume that node $n_{i}$ has received a message $M_{A}^{\prime}(j)$.

- If node $n_{i}$ is the destination of $l_{d s t}$, it starts process D. If not, it forwards the message to an applicable node for $l_{d s t}$.

\subsubsection{Process $D$}

Assume that node $n_{i}$ has received a message $M_{A}^{\prime}(j)$.

- If node $n_{i}$ does not know the value of $S_{\hat{K}_{R_{I D}}^{-1}}\left(H\left(T_{c}\right)\right)$, it suspends this process until it receives a message $M_{A}^{\prime}$.

- Node $n_{i}$ calculates $F_{L}\left(S_{\hat{K}_{R_{I D}}^{-1}}\left(H\left(T_{c}\right)\right), j\right)$ and checks whether or not the calculated value is the same as the value of $l_{d s t}$.

- Node $n_{i}$ calculates a public key of the node whose node ID is $j ; K_{j}=F_{E}(j)$, and calculates $H\left(T_{c}\|j\| l_{j}\right)$. It then verifies $S_{K_{j}^{-1}}\left(H\left(T_{c}\left\|n_{j}\right\| l_{j}\right)\right)$.

- Node $n_{i}$ saves the message $M_{A}^{\prime}(j)$. If in this internal turn it receives another message $M_{A}^{\prime}(j)$ which contains node ID $j$, it checks whether or not $l_{j}$ is also the same. If it is not the same, it concludes that it detected a replication attack. It broadcasts these two messages to all the nodes in the network.

\subsection{ANALYSIS}

If an attacker compromises some nodes, he may learn the locations of witness nodes from them. To learn the locations of witness nodes, he needs to compromise the first node of a group if no nodes are broken. We call a turn in which an attacker compromises the first node of a group a compromised turn. If an attacker knows the locations of witness nodes of several consecutive turns, he can perform replicated node attacks from the first compromised turn of the sequence of compromised turns to the last compromised turn of the sequence. Therefore, we need to analyze the expected maximum number of consecutive compromised turns for the number of compromised nodes.

We also need to analyze another feature. If some of the nodes are faulty (i.e., compromised or broken), some turns may not be able to start a detection process. We call a turn that cannot start the detection process a faulty turn. We analyze the expected average number of consecutive faulty turns.

\subsubsection{Maximum number of consecutive compromised} turns.

Assume that $C_{n}$ nodes are compromised. The probability that number of groups of which the first node compromised is $C_{G}$ is

$$
P_{c}\left(N, N_{G}, C_{n}, G_{c}\right)=\frac{\left(\begin{array}{c}
N_{G} \\
C_{G}
\end{array}\right)\left(\begin{array}{c}
N-N_{G} \\
C_{n}-C_{G}
\end{array}\right)}{\left(\begin{array}{c}
N \\
C_{n}
\end{array}\right)}
$$

Each group has an ID. The ID of the first group is 1, and the ID of the last group is $N_{G}$.

We require the expected number of maximum consecutive compromised groups. For example, if an attacker compromises nodes in groups $3,4,5,7$, and 10 , the number of maximum consecutive compromised groups is three (groups $3,4,5$ ). Here, the next group of group $N_{G}$ is group 1. The maximum number of consecutive compromised groups is the maximum number of turns in which 
the attacker can learn the locations of witness nodes from compromised nodes. We assume that the number of executing the detection processes is $V$.

First, we require the number of combinations such that the maximum number of consecutive compromised groups is equal to $M_{S}$ when an attacker compromises $C_{G}$ groups from $N_{G}$ groups. Here, let us assume that the next group of group $N_{G}$ is nonexistent. The number of combinations $f_{s c}\left(N_{G}, C_{G}, M_{S}\right)$ is

$$
\begin{aligned}
& f_{s c}\left(N_{G}, C_{G}, M_{S}\right) \\
& =\left\{\begin{array}{l}
\text { if }\left(C_{G}=0 \& M_{S}=0\right) \| N_{G}=C_{G}=M_{S} \text { then } 1, \\
\text { else if } N_{G} \leq 0\left\|N_{G}<C_{G}\right\| C_{G}<M_{S} \text { then } 0, \\
\text { else } \sum_{i=0}^{M_{S}} f_{s c}\left(N_{G}-i-1, C_{G}-i, M_{S}\right) \\
+\sum_{i=0}^{M_{S}-1} f_{s c}\left(N_{G}-M_{S}-1, C_{G}-M_{S}, i\right) .
\end{array}\right.
\end{aligned}
$$

We can get the expected number of maximum consecutive compromised groups when an attacker compromises $C_{g}$ groups from $N_{G}$ groups. Here, let us assume that the next group of group $N_{G}$ is group 1. The expected number of maximum consecutive compromised groups $E_{m}\left(N_{G}, C_{G}\right)$ is

$$
\begin{aligned}
& E_{m}\left(N_{G}, C_{G}\right)=\frac{1}{\left(\begin{array}{l}
N_{G}-1 \\
C_{G}-1
\end{array}\right)} \times \\
& \left\{\begin{array}{l}
\text { if } N_{G}=C_{G} \text { then } V, \\
\text { else }\left(C_{G}\right)^{2} \\
C_{G}-1 \\
+\sum_{j=1}^{C_{G}} \sum_{i=1}\left[j \cdot \max (i, j) \cdot f_{s c}\left(N_{G}-j-2, C_{G}-j, i\right)\right] .
\end{array}\right.
\end{aligned}
$$

Finally, we can get the expected number of maximum consecutive compromised turns when an attacker compromises $C_{n}$ nodes from equations 1 and 3 .

$$
E_{M}\left(N, N_{G}, C_{n}\right)=\sum_{i=1}^{C_{n}} P_{c}\left(N, N_{G}, C_{n}, i\right) \cdot E_{m}\left(N_{G}, i\right) .
$$

\subsubsection{Average number of consecutive faulty turns.} Assume that $F_{n}$ nodes are faulty (broken or compromised) of $N$ nodes. The probability that the number of groups whose nodes are all faulty is $F_{g}$ is

$$
\begin{aligned}
& P_{f}\left(N, N_{G}, F_{n}, F_{g}\right) \\
& =\frac{\left(\begin{array}{c}
N_{G} \\
F_{g}
\end{array}\right) C_{f}\left(N_{G}-F_{g}, N / N_{G}, F_{n}-F_{g} \cdot\left(N / N_{G}\right)\right)}{\left(\begin{array}{c}
N \\
F_{n}
\end{array}\right)},
\end{aligned}
$$

where $C_{f}\left(N_{G}, N_{p}, F_{n}\right)$

$$
=\left\{\begin{array}{l}
\text { if } F_{n}=0 \text { then } 0, \\
\text { else }\left(\begin{array}{c}
N_{G} \cdot N_{p} \\
F_{n}
\end{array}\right) \\
-\sum_{i=1}^{\min \left(N_{G}, F_{n} / N_{p}\right)} N_{G} C_{i} \cdot C_{f}\left(N_{G}-i, N_{p}, F_{n}-i \cdot N_{p}\right) .
\end{array}\right.
$$

When the number of fault turns is $F_{g}$, the average number of consecutive faulty turns is

$$
E_{f}\left(N_{G}, F_{g}\right)=\left\{\begin{array}{l}
\text { if } N_{G}=F_{g} \text { then } V, \\
\text { else } \sum_{j=0}^{F_{g}} j \cdot\left[\frac{N_{G}-F_{g}}{N_{G}-j} \prod_{i=0}^{j-1} \frac{F_{g}-i}{N_{G}-i}\right] .
\end{array}\right.
$$

Finally, we can get the expected number of average consecutive faulty turns when $F_{n}$ nodes from equations 5 and 6 .

$$
\begin{aligned}
& E_{F}\left(N, N_{G}, F_{n}\right) \\
& =\sum_{i=1}^{\min \left(N_{G}, F_{n} \cdot N_{G} / N\right)} P_{f}\left(N, N_{G}, F_{n}, i\right) \cdot E_{f}\left(N_{G}, i\right) .
\end{aligned}
$$

\section{EVALUATION}

We conduct mathematical analysis and simulations of our protocols.

\subsection{Mathematical analysis}

We conduct mathematical analyses of the detection process (1. maximum number of consecutive compromised turns and 2. average number of consecutive faulty turns) and our reporter node determination protocol. We set the number of nodes of a network to 10,000 .

\subsubsection{Maximum number of consecutive compromised turns.}

Figure 3 represents the relationship among the number of compromised nodes $\left(C_{n}\right)$, the number of nodes of a group $\left(N_{p}\right)$, and the expected maximum number of consecutive compromised turns $\left(E_{M}\right)$. In Fig. 3, the number of the detection processes $V$ was set to 10, and in Fig. 3, $V$ was set to 1,000. Assume that an attacker compromises more than $N_{G}$ nodes. Although the probability that the number of compromised turns $\left(E_{M}\right)$ is equal to $N_{G}$ is very small, it is not zero. If $E_{M}$ is equal to $N_{G}$, the attacker can learn all witness nodes in all turns. Therefore, $N_{G}$ should not be very 


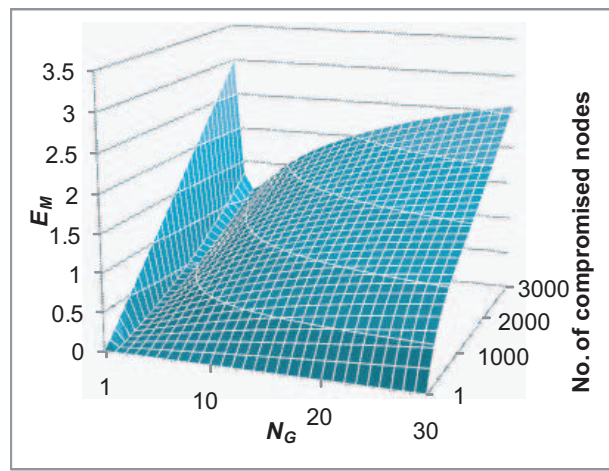

(a) $V=10$

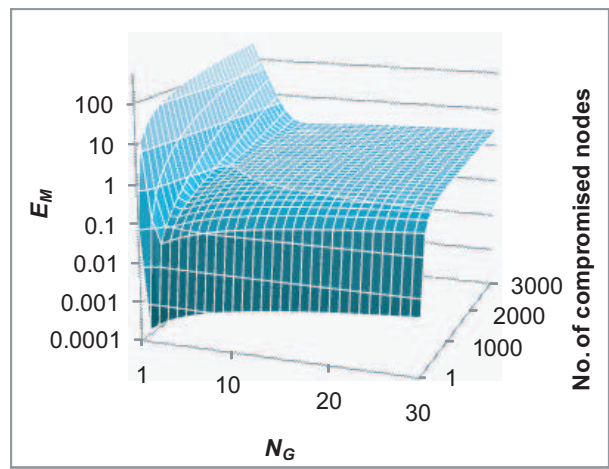

(b) $V=1,000$

Figure 3: Expected maximum no. of consecutive compromised turns $(N=10,000)$

small. If $V$ is greate, a relatively greater $N_{G}$ is better. However, $N_{G}$ should not also be very greate value. If $N_{G}$ is greate, the number of nodes per group is small. Therefore, the probability that an attacker compromises the first node of a group increases.

We know from the Fig. 3 that $E_{M}$ is only $1.0-1.7$ even if the attacker compromises 3,000 of 10,000 nodes.

\subsubsection{Average number of consecutive faulty turns.}

Figure 4 represents the relationship among the number of faulty nodes $\left(F_{n}\right)$, the number of groups $\left(N_{G}\right)$, and the expected average number of consecutive faulty turns $\left(E_{F}\right)$. In Fig. 4, $V$ was set to 100 . We conducted analyses of varying $V$ from 10 to 1,000 , however, the results are almost the same as each other. To reduce $E_{F}$, we know from the figure that $N_{G}$ should be small. This is because a faulty turn means a turn whose $N / N_{G}$ nodes are all faulty. Even if 5,000 of 10,000 nodes are faulty, the number of consecutive faulty turns remains very small.

\subsection{Simulations}

We conducted simulations to demonstrate the effectiveness of our detection protocol of replicated node attacks. Table 2 lists the re-

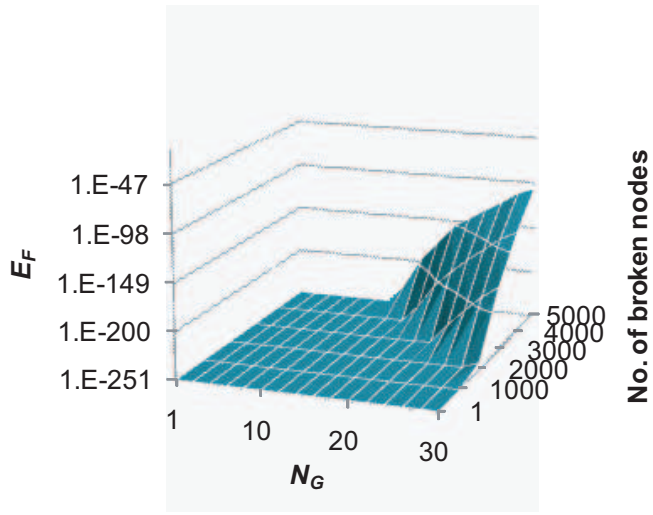

Figure 4: Expected average no. of consecutive faulty turns $(N=10,000, V=100)$

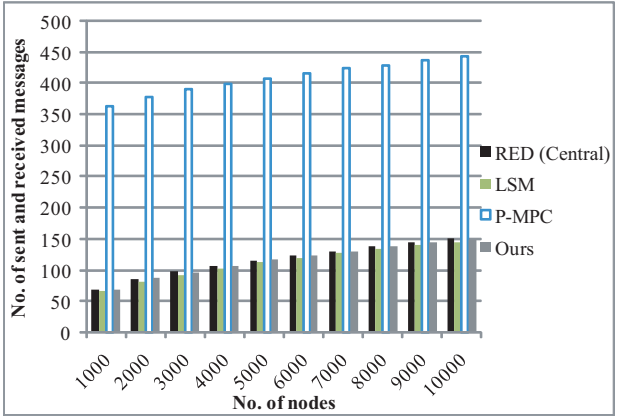

Figure 5: Communication overhead

sources required by RED [23], LSM [15], P-MPC [5], and our protocol. RED uses a central trusted entity (e.g., a base station). The authors of [15] proposed the LSM and RM protocols. Because LSM is a modification of RM, we only compared LSM with our protocol. By the same token, the authors of [5] proposed the SDC and P-MPC protocols, but we only compared P-MPC.

The number of nodes was 10,000. The orders in Table 2 are theoretical values. The numeric values represent the simulation results. The values of $s$ and $p_{s}$ in the P-MPC protocol are the protocol's system parameters. We used the default values for the related studies if they had system parameters. The results of our protocol are almost the same as that of RED. In our protocol, each node must do a signature check and save $S_{\hat{K}_{R_{I D}}^{-1}}\left(H\left(T_{c}\right)\right)$. Therefore, our protocol needs one more memory occupancy and signature check compared with RED.

Next, we conducted a simulation of communication overhead. Figure 5 shows the results. We varied the number of nodes from 1,000 to 10,000 . We found that LSM needed a large overhead and the other protocols had almost equal overheads.

Next, we conducted a simulation of the detection rate with no compromised nodes. The results are shown in Fig. 6. We know that the detection rates of RED and ours are almost the same. That of PMPC was a little smaller than ours, and that of LSM was relatively 
Table 2: Comparison with related work

\begin{tabular}{l|l|l|l|l|l}
\hline \hline & Memory Occupancy & Sent Message & Received Message & Signature Check & Detection rate \\
\hline RED (Central) & $O(r)$ & $O(r \cdot \sqrt{N})$ & $O(r \cdot \sqrt{N})$ & $O(r)$ & \\
& 1.00 & 20.49 & 49.35 & 6.02 & 0.914 \\
\hline LSM & $O(r \cdot \sqrt{N})$ & $O(r \cdot \sqrt{N})$ & $O(r \cdot \sqrt{N})$ & $O(r \cdot \sqrt{N})$ & \\
& 19.62 & 20.55 & 48.36 & 12.52 & 0.338 \\
\hline P-MPC & $O\left(r \cdot s \cdot p_{s}\right)$ & $O(r \cdot \sqrt{N})+O(s)$ & $O(r \cdot \sqrt{N})+O(s)$ & $O\left(r \cdot s \cdot p_{s}\right)$ & \\
& 7.60 & 167.21 & 195.62 & 13.62 & 0.866 \\
\hline Ours & $O(r)$ & $O(r \cdot \sqrt{N})$ & $O(r \cdot \sqrt{N})$ & $O(r)$ & \\
& 2.00 & 20.67 & 48.59 & 7.00 & 0.912 \\
\hline
\end{tabular}

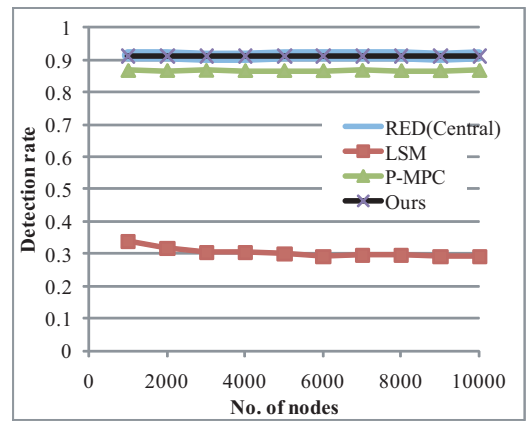

Figure 6: Detection rate with no compromised nodes

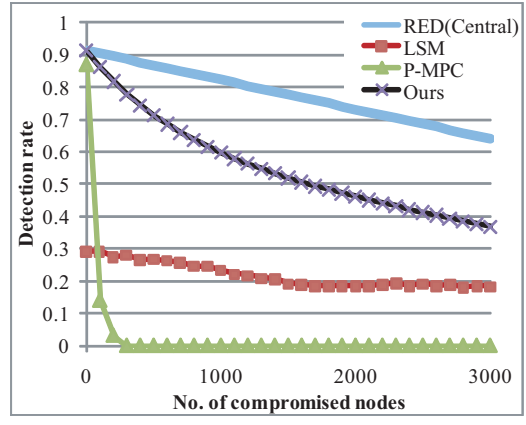

Figure 7: Detection rate depending on no. of compromised nodes

small compared with other protocols.

Finally, we conducted a simulation of the detection rate when the number of compromised nodes varied. If a witness node was a compromised node, the replicated node attack was not detected. In our protocol, the detection also failed if the attacker knew the location of the witness nodes. We set the number of nodes to 10,000 . RED, LSM, and our protocols had high resiliency to many compromised nodes. However, P-MPC was easily affected by compromised nodes because all witness nodes candidates are leaked to an attacker if he compromises one node.

\section{CONCLUSION}

We proposed a detection protocol for node replicated attacks. We realized full distributed detection by assigning the process initial- ization role to each node. Each node is preloaded with a unique seed and uses it to initialize the detection process. We divide nodes into groups to increase resiliency to many compromised nodes, and we verified the resiliency of our protocol in mathematical analyses and in simulations. We also proposed a reporter node determination protocol. This protocol can be used for our detection protocol and other detection protocols dealing with node replication attacks. By using this protocol, the detection rate of node replication attacks was significantly higher than that of existing node determination protocols when there were several compromised nodes.

\section{REFERENCES}

[1] Dan Boneh, Craig Gentry, and Michael Hamburg. Space-efficient identity based encryptionwithout pairings. In IEEE FOCS, pages 647-657, 2007.

[2] Jehoshua Bruck, Jie Gao, and Anxiao (Andrew) Jiang. Localization and routing in sensor networks by local angle information. In ACM MOBIHOC, pages 181-192, 2005.

[3] Haowen Chan, Adrian Perrig, and Dawn Song. Random key predistribution schemes for sensor networks. In IEEE Symposium on Security and Privacy, pages 197-213, 2003.

[4] Gilbert Chen, Joel W. Branch, and Boleslaw K. Szymanski. Local leader election, signal strength aware flooding, and routeless routing. In IEEE IPDPS, page 244.1, 2005.

[5] M. Conti, R. Di Pietro, L.V. Mancini, and A. Mei. A randomized, efficient, and distributed protocol for the detection of node replication attacks in wireless sensor networks. In ACM MobiHoc, pages 80-89, 2007.

[6] Dezun Dong, Yunhao Liu, and Xiangke Liao. Self-monitoring for sensor networks. In ACM MobiHoc, pages 431-440, 2008.

[7] Wenliang Du, Jing Deng, Yunghsiang S. Han, Shigang Chen, and Pramod K. Varshney. A key management scheme for wireless sensor networks using deployment knowledge. In IEEE INFOCOM, 2004.

[8] Brad Karp and H. T. Kung. GPSR: Greedy perimeter stateless routing for wireless networks. In ACM MOBICOM, pages 243-254, 2000.

[9] Jooyoung Lee and Douglas R. Stinson. On the construction of practical key predistribution schemes for distributed sensor networks using combinatorial designs. ACM Trans. Inf. Syst. Secur., 1(2):1094-9224, 2008.

[10] Donggang Liu, Peng Ning, and Wenliang Du. Group-based key predistribution for wireless sensor networks. ACM Trans. Sen. Netw., 4(2):1550-4859, 2008.

[11] Liqian Luo, Chengdu Huang, Tarek F. Abdelzaher, and Jack Stankovic. Envirostore: A cooperative storage system for disconnected operation in sensor networks. In IEEE INFOCOM, pages 1802-1810, 2007. 
[12] Sergio Marti, T. J. Giuli, Kevin Lai, and Mary Baker. Mitigating routing misbehavior in mobile ad hoc networks. In ACM MOBICOM, pages 255-265, 2000.

[13] James Newsome, Elaine Shi, Dawn Song, and Adrian Perrig. The sybil attack in sensor networks: analysis \& defenses. In ACM IPSN, pages 259-268, 2004.

[14] Peng Ning, An Liu, and Wenliang Du. Mitigating dos attacks against broadcast authentication in wireless sensor networks. ACM Trans. Sen. Netw., 4(1):1-35, 2008.

[15] Bryan Parno, Adrian Perrig, and Virgil Gligor. Distributed detection of node replication attacks in sensor networks. In IEEE S\&P, pages 49-63, 2005.

[16] Adrian Perrig, Robert Szewczyk, Victor Wen, David E. Culler, and J. D. Tygar. SPINS: Security protocols for sensor networks. In ACM MOBICOM, pages 189-199, 2001.

[17] Kui Ren, Kai Zeng, and Wenjing Lou. Secure and fault-tolerant event boundary detection in wireless sensor networks. IEEE Transactions on Wireless Communications, 7:354-363, 2008.

[18] Adi Shamir. Identity-based cryptosystems and signature schemes. In CRYPTO 84 on Advances in cryptology, pages 47-53, 1985.

[19] Radu Stoleru, Pascal Vicaire, Tian He, and John A. Stankovic. Stardust: A flexible architecture for passive localization in wireless sensor networks. In ACM SENSYS, pages 57-70, 2006.

[20] Hailun Tan, Sanjay Jha, Diet Ostry, John Zic, and Vijay Sivaraman. Secure multi-hop network programming with multiple one-way key chains. In ACM WiSec, pages 183-193, 2008.

[21] Anthony D. Wood and John A. Stankovic. Denial of service in sensor networks. IEEE Computer, 35(10):54-62, 2002.

[22] Hao Yang, James Shu, Xiaoqiao Meng, and Songwu Lu. SCAN: Self-organized network layer security in mobile ad hoc networks. IEEE Journal on Selected Areas in Communications, 24(2):261-273, 2006.

[23] Bo Zhu, Venkata Gopala Krishna Addada, Sanjeev Setia, Sushil Jajodia, and Sankardas Roy. Efficient distributed detection of node replication attacks in sensor networks. In IEEE ACSAC, pages 257-267, 2007. 